\title{
A Study on the Prevalence and Virulence of Avian Influenza A (H5N1, H5N6 and H7N9) Viruses on Poultry at Lang Son Markets in Vietnam
}

\author{
Dang Xuan Binh ${ }^{1,}$, Nguyen Thi Thu $\mathrm{Ha}^{2}$ \\ ${ }^{1}$ Faculty of Animal Husbandry and Veterinary Medicine, Thai Nguyen University of Agriculture and Forestry, Thai Nguyen City, Vietnam \\ ${ }^{2}$ Sub Department of Animal Health, Department of Agriculture and Rural Development, Lang Son City, Lang Son Province, Vietnam
}

\section{Email address:}

binhdx@tnu.edu.vn (D. X. Binh)

${ }^{*}$ Corresponding author

\section{To cite this article:}

Dang Xuan Binh, Nguyen Thi Thu Ha. A Study on the Prevalence and Virulence of Avian Influenza A (H5N1, H5N6 and H7N9) Viruses on Poultry at Lang Son Markets in Vietnam. Animal and Veterinary Sciences. Vol. 4, No. 4, 2016, pp. 52-61. doi: 10.11648/j.avs.20160404.12

Received: June 12, 2016; Accepted: August 23, 2016; Published: August 25, 2016

\begin{abstract}
In 2014 and 2015, composite cloacal and tracheal swab samples of poultry sold at markets in Lang Son province (Vietnam) were collected in order to examine the circulation of avian influenza A (H5N1, H5N6 and H7N9) viruses. The results show that: (i) for the chicken illegally imported from China, $3.93 \%$ to $6.43 \%$ of the chickens were found positive with type A, $3.18 \%$ to $3.78 \%$ were positive with subtype $\mathrm{H} 5$, and $0.37 \%$ to $3.40 \%$ were positive with subtype N1 viruses; (ii) for the chicken sold at local markets, $15.55 \%$ were found positive with type A, $5 \%$ were positive with subtype H5, and $2.77 \%$ were positive with subtype N1 viruses; (iii) for the chicken sold at the border markets, $13.49 \%$ were positive with type A, $4.76 \%$ were positive with subtype H5, and 3.96\% were positive with subtype N1 viruses; (iv) for the chicken and ducks sold at the border markets with avian influenza A viruses (H5N6), $10.4 \%$ to $36.1 \%$ were positive with type A; $4.1 \%$ to $6.25 \%$ were positive with subtype $\mathrm{H} 5$; and $2.3 \%$ to $6.25 \%$ were found positive with subtype N6 viruses; (v) subtype H7N9 were not prevalent on the poultry sold at the border and local markets; and (vi) laboratory chicken for testing IVPI (Intravenous Pathogenicity Index) showed symptoms of sickness and died within one to two days after infected with H5N1 and H5N6 viruses. The IVPI ranged from 2.9 to 2.92 . This shows that the avian influenza virus strains isolated in Lang Son were highly virulent (HPAI).
\end{abstract}

Keywords: Avian Influenza, Type A, H5N1, H5N6, H7N9, IVPI

\section{Introduction}

Avian influenza is a highly contagious viral disease affecting the respiratory, digestive and/or nervous system of a number of bird species. It is caused by influenza A viruses. There are two types of avian influenza viruses. These are called low pathogenic (LPAI) and highly pathogenic (HPAI), depending on the severity of the disease that they cause in birds, with high infection and dead rates on poultry within 24-48 hours of being infected [1], [2], [4], [7], [8]. The disease is categorized inthe A list of the most dangerous communicable diseases by World Organisation for Animal Health. The disease has been found in Vietnam, its neighbouring countries and many other countries in the world [4], [7], [10], [16].
Avian influenza A viruses (H5N1, H5N6...) cause diseases on poultry (chickens, ducks, geese, and birds) [2], [6], [11], [14], [15] and could be transmitted to human, which cause serious damages on the society and economies. In 2014, ten countries including Vietnam and China announced the prevalence of avian influenza. In the same year, it was announced that avian influenza A, H5N6 occured in China and Laos [1], [8], [10], [16].

Being at the center of trade and economic transactions of the North and of Vietnam, Lang Son province has witnessed avian influenza outbreaks in various areas in different periods of time. The outbreaks caused great damages to the agriculture sector. In August 2014, Lang Son was the first province in Vietnam to announce the prevalence of avian influenza type A/H5N6. 
In 2015, type A/H5N1 influenza epidemic occurred in 23 nations and territories including China and Vietnam; in the same year, avian influenza type A/H5N6 occurred in China, Hongkong (China), Laos, and Vietnam. Similar to what happened in 2014, in 2015, in China, there were 225 people infected with influenza type A/H7N9, 94 of them died. Regarding causing diseases on human, in 2014, there were 311 cases of $\mathrm{A} / \mathrm{H} 7 \mathrm{~N} 9$ infection and 130 of the patients died. Up to now, H7N9 viruses have only been found on human and poultry in China [11], [14], [15].

Lang Son is a border mountainous province in Northeast Vietnam, with a number of national road running through; the north shares the same border with Chongzuo (Quangxi, China) with $253 \mathrm{~km}$ of borderline; two international and two national border gates, seven border markets and numerous local markets, which create favourable conditions for the province to become an important trade center of Vietnam and China, through which goods flow to the Middle East and European countries.

With the complex spread of avian influenza epidemics in Vietnam and a relatively high number of infected population, which tends to get higher and higher, and with the predictions about the possibility of the occrence of the transformed virus strains which can cause a serious avian influenza epidemics on human, the project entitled "A Study on the Prevalence and Virulence of Avian influenza A (H5N1, H5N6 and H7N9) viruses on Poultry at Lang Son Markets in Vietnam" was carried out in order to proactively respond to the epidemics.

\section{Materials and Methods}

\subsection{Contents}

- Identification of sites for examining the prevalence of avian influenza A viruses, subtype H5N1.

- Examination of the chicken illegally imported from China for avian influenza A viruses, subtype H5N1.

- Determination of the prevalence of avian influenza A viruses, subtype $\mathrm{H} 5 \mathrm{~N} 1$ on chicken sold at some local markets.

- Determination of the prevalence of avian influenza A viruses, subtype $\mathrm{H} 5 \mathrm{~N} 1$ on ducks sold at three border markets.

- Examination of the fecal matter and waste collected at the poultry selling points for infection of avian influenza A viruses, subtype H5N1, H5N6, and subtype H7N9.

- Assessment of the virulence (IVPI) of the of the avian influenza virus strains H5N1 and H5N6.

\subsection{Cooperation}

- Lang Son Sub Department of Animal Health collected the samples.

- National Centre for Veterinary Diagnosis and Regional Animal Health Office No. II examined the samples.

- Centers for Disease Control and Prevention (CDC, USA) through Food and Agriculture Organization (FAO) and "Vietnam Avian and Human Influenza Control and Preparedness Project in Lang Son province” (VAHIP) provided technical, facility, and financial supports.

\subsection{Study Area}

- Lang Son Sub Department of Animal Health collected the samples.

- Avian influenza A viruses, subtype H5N1, H5N6, and H7N9 on poultry (chicken and ducks) in Lang Son province, Vietnam.

- Samples of medical waste include cloacal and tracheal swab samples of poultry (chicken and ducks) and environmental swab samples (waste water from, and fecal matter of poultry) collected in Lang Son province (local poultry and poultry illegally imported from China).

- Materials, chemicals, and RT-PCR equipment at National Centre for Veterinary Diagnosis (Ha Noi) and Regional Animal Health Office No. II (Hai Phong) were used for examining for avian influenza A viruses, subtypes H5N1, H5N6, and H7N9.

\subsection{Time Duration}

- From 2014 to 2015

\subsection{Methods}

Multi-stage random cluster sampling was used. Cloacal and tracheal swab samples and environmental swab samples (waste water and waste matter) were collected at poultry buying points or markets.

Poultry dealers were interviewed to collect necessary information.

Update the collected information to the tables and indexes.

* Swab samples examination process

Step 1: Process and encode the samples

Step 2: Separate the samples manually or automatically

Step 3: Prepare the master mixes for RT-PCR

Step 4: Prepare ARN samples and the mixes in Step 3, put them to the RT-PCR machine

After the samples were found positive with avian influenza type A, started examining for $\mathrm{H} 5$

After the samples were found positive with H5, started examining for $\mathrm{N} 1$ and $\mathrm{N} 6$

After the samples were found positive with $\mathrm{H} 7$, started examining for N9

* Cloacal and tracheal swab sample collection

Sterized cotton buds were used to get cloacal and tracheal swab samples on mature poultry. A cotton bud was used for gettting one sample on a chicken (01 single sample). After that, each 05 single samples formed a composite sample which was stored in sample containers with preserving fluid.

* Environmental swab sample collection

Sterized cotton buds were used to collect environmental swab samples: fecal matter onthe surfaces of the poultry cages and on the ground, waste water, and thewater fed to the poultry in the containers (all of these are called environmental waste). In details:

The water fed to the poultry: 10 single samples (02 composite samples). 
Poultry fecal matter on the surfaces of the poultry cages: 10 single samples ( 02 composite samples).

Table 1. Primers for identifying the prevalence of avian influenza A viruses.

\begin{tabular}{lllll}
\hline Types of viruses & Gene fragment & Size & Primers & Primer sequence (5'-->3') \\
\hline \multirow{2}{*}{ Type A } & \multirow{2}{*}{ Matrix (M) } & $244 \mathrm{bp}$ & M30F2/08 & ATGAGYCTTYTAACCGAGGTCGAAACG \\
& & M264R3/08 & TGGACAAANCGTCTACGCTGCAG \\
\hline
\end{tabular}

Table 2. Primers for indentifying the prevalence of subtype H5.

\begin{tabular}{lllll}
\hline HA subtype & Primer & Gene fragment & Size & Primer sequence (5'-->3') \\
\hline H5 & H5-918F & \multirow{2}{*}{69277} & $249 \mathrm{bp}$ & CCARTRGGKGCKATAAAYTC \\
& H5-1166 & & & KGTCTGCWGCRTAYCCRCTY \\
H7 & H7-937F & M31689 & $241 \mathrm{bp}$ & ATYAAYMSYAGRRCWGTRGG \\
& H7-1177 & & GATCWATTGCHGAYTGRGTG \\
\hline
\end{tabular}

Table 3. Primers for indentifying the prevalence of subtypes N1, N6 and N9.

\begin{tabular}{lllll}
\hline NA subtype & Primer & Gene fragment & Size & Primer sequence (5'-->3') \\
\hline \multirow{2}{*}{ N1 } & N1-54F & \multirow{2}{*}{ CY003931 } & $245 \mathrm{bp}$ & TCARTCTGYATGRYAAYTGG \\
& N1-298R & & & GGRCARAGAGAKGAATTGCC \\
N6 & N6-57F & \multirow{2}{*}{ CY005464 } & \multirow{2}{*}{$264 \mathrm{bp}$} & AGGAATGACACTATCSGTAGTAAG \\
N6-307R & & \multirow{2}{*}{ GAYAGRATRTGCCATGAGTTYAC } \\
N9 & N9-64F & CY004701 & GTAATAGGCACRATYGCAGT \\
& N9-290R & & CCTTTRGTYARRTTATTGAA \\
\hline
\end{tabular}

* Intravenous Pathogenicity Index Assessment

The assessment of the virulence of the viruses was performed on laboratory using Intravenous Pathogenicity Index (IVPI).

The experiment was performed on ten six-week-old Egyptian chicken (Fayoumi) which had been identified as carrying no antibody for avian influenza viruses H5N1.

Amniotic fluid was infected with antibody titer HA $>1 / 16$, diluted in sterile Sodium chloride substance with the ratio of $1 / 10$. $0.1 \mathrm{ml}$ of the diluted fluid was injected into the vein of the laboratory chicken. The laboratory chicken was examined once every 24 hours in 10 days. The chicken were then given a point, with $0=$ Normal, $1=$ Sick, $2=$ Very sick, and $3=$ Dead. (The states of being Sick or Very sick were assessed clinically. The "Sick" ones would normally show one of the following symptoms, and the "Very sick" ones would show more than one of them: respiratory problems, being doleful, diarrhea, the uncovered skin areas or the combs get pale, swollen face and/or head, and symptoms of neurological disorder. Dead chicken will be marked with point 3 for all the remaining days of the experiment)

IVPI was the average point of the average point of each chicken observed in ten days. If the index figure is 3.00 , it means all the chicken died within 24 hours. If the index figure is 0.00 , it means no observed chicken showed clinical symptoms in the ten days. Avian influenza viruses have IVPI $>1.2$, which means the viruses are highly virulent [7], [12], [14], [15].

\section{Results and Discussion}

\subsection{Research Sites Identification}

A portable Pocket Global Positioning System (GPS) with the national frame of VN 2000 was used for identifying the sites for collecting the samples to examine the prevalence of Avian influenza A viruses, H5N1 in Lang Son. The results are presented in Table 4.

Table 4. Sites identified by GPS for sample collection.

\begin{tabular}{|c|c|c|c|}
\hline \multirow{2}{*}{ STT } & \multirow{2}{*}{ Sample collection sites } & \multicolumn{2}{|c|}{ Positions idenfified by GPS } \\
\hline & & $\mathbf{X}$ & $\mathbf{Y}$ \\
\hline 1 & Poultry buying points in Tran Khanh Du (Gieng Vuong) & 48Q0681888 & UMT2418165 \\
\hline 2 & Poultry buying points in Vuon Sai (Lang Son city) & 48Q0675494 & UMT2428441 \\
\hline 3 & Dong Dang market & 48Q0675131 & UMT2428316 \\
\hline 4 & Poultry buying points in Cao Loc & 48Q0682518 & UMT2419356 \\
\hline 5 & Poultry buying points in Tam Thanh & 48Q0680100 & UMT2417154 \\
\hline 6 & Ky Lua market & 48Q0681679 & UTM 2418263 \\
\hline 8 & Poultry buying points in Hop Thanh (That Khe) & 48Q0684249 & UMT2417796 \\
\hline 9 & Poultry buying points in Na Duong, Loc Binh & 48Q0703466 & UMT2400021 \\
\hline 10 & Dong Mo market & 48Q0663273 & UMT2395987 \\
\hline 11 & Poultry buying points in Dinh Thi Hang (Lang Son city) & 48Q0680839 & UMT2416470 \\
\hline 12 & Poultry buying points in Vy Thanh Chung (Lang Son city) & 48Q0682255 & UMT2417150 \\
\hline 13 & Chi Lang market & 48Q0681641 & UMT2416730 \\
\hline 14 & Poultry buying points in Linh Thi Tuyet (Met townlet) & 48Q0681795 & UMT2418303 \\
\hline
\end{tabular}


Table 4 shows that: the $\mathrm{X}$ and $\mathrm{Y}$ positions of the medical waste sample collection sites including poultry buying points (there were 10 of them) and samples collection sites at markets in Lang Son province (05 markets). This would serve as a scientific base for developing an epidemiology map on examining the prevalence of avian influenza viruses in Lang Son province.

\subsection{Avian Influenza A Viruses, Subtype H5N1 on Illegally Imported Chicken}

In 2014 and 2015, cloacal and tracheal swab samples were collected on mature chicken illegally imported from China to Lang Son (Viet Nam) which was captured by Vietnamese authorities. The results are presented in Table 5.

Table 5 shows that: In 2014, 264 composite cloacae and tracheal swab samples of mature chicken illegally imported from China to Lang Son province were collected to examine the prevalence of avian influenza A viruses, subtype H5N1. In details: In March: 23 composite samples were collected, 2 were found positive with avian influenza A viruses (accounting for $8.69 \%$ ), of which 1 were found positive with subtype H5 (accounting for 4.34\%), but none were found positive with subtype N1; In April: examined 46 samples, In May: 14 samples were examined, In June: 12 samples were examined but none were found positive with avian influenza viruses A/H5N1; In August: examined 42 samples, 2 were found positive with avian influenza A viruses (accounting for $4.76 \%$ ), but none were found positive with subtype H5; In September: 24 samples were examined, 3 were found positive with avian influenza A viruses, and 1 were found positive with subtype H5N1 (accounting for 4.16\%); In October: 50 samples were examined, 7 were found positive with avian influenza A viruses (accounting for 14\%), and 6 were found positive with subtype $\mathrm{H} 5 \mathrm{~N} 1$ (accounting for 12\%); In November: 53 samples were examined, 3 were found positive with avian influenza A viruses (accounting for $5.66 \%$ ), of which 2 were found positive with subtype H5N1 (accounting for $3.77 \%$ ). In January, February, July, and December there were no samples examined due to the fact that no illegally imported chicken from China to Vietnam were captured by Vietnamese authorities. This finding was similar to that of [9] and [13].

Table 5. Avian influenza A viruses, subtype H5N1 on illegally imported chicken.

\begin{tabular}{|c|c|c|c|c|c|c|c|}
\hline \multirow{3}{*}{ Month } & \multirow{3}{*}{ No. of samples (Composite swabs) } & \multicolumn{6}{|c|}{ Results } \\
\hline & & \multicolumn{2}{|c|}{ Type A } & \multicolumn{2}{|c|}{ Subtype H5 } & \multicolumn{2}{|c|}{ Subtype N1 } \\
\hline & & $(+)$ & $\%$ & $(+)$ & $\%$ & $(+)$ & $\%$ \\
\hline \multicolumn{8}{|l|}{ In 2014} \\
\hline 3 & 23 & 2 & 8.69 & 1 & 4.34 & 0 & 0 \\
\hline 4 & 46 & 0 & 0 & 0 & 0 & 0 & 0 \\
\hline 5 & 14 & 0 & 0 & 0 & 0 & 0 & 0 \\
\hline 6 & 12 & 0 & 0 & 0 & 0 & 0 & 0 \\
\hline 9 & 24 & 3 & 12.5 & 1 & 4.16 & 1 & 4.16 \\
\hline 10 & 50 & 7 & 14.0 & 6 & 12.0 & 6 & 12.0 \\
\hline 11 & 53 & 3 & 5.66 & 2 & 3.77 & 2 & 3.77 \\
\hline \multicolumn{8}{|l|}{ In 2015} \\
\hline 2 & 12 & 2 & 16.66 & 1 & 8.33 & 1 & 8.33 \\
\hline 3 & 98 & 5 & 5.1 & 3 & 3.06 & 1 & 1.02 \\
\hline 4 & 111 & 3 & 2.7 & 2 & 1.8 & 0 & 0 \\
\hline
\end{tabular}

In 2015, 233 composite cloacal and tracheal swab samples of mature chicken illegally imported from China were collected to examine the prevalence of avian influenza A viruses subtype H5N1, In details: In February, 12 composite samples were collected, of which 2 were found positive with avian influenza A viruses (accounting for $16.66 \%$ ), of which 1 were found positive with subtype $\mathrm{H} 5 \mathrm{~N} 1$ (accounting for 8.33\%); In March: examined 98 samples, 5 were found positive with avian influenza A viruses (accounting for $5.1 \%$ ), 3 were found positive with subtype H5 (accounting for $3.06 \%$ ), 1 were found positive with subtype N1 (accounting for 1.02\%); In April: examined 111 samples, 3 were found positive with avian influenza A viruses (accounting for 2.7\%), 2 were found positive with subtype H5 (accounting for $1.8 \%$ ), but none were found positive with subtype N1; In December: examined 12 samples, 11 were found positive with avian influenza A viruses (accounting for $91.66 \%$ ), 11 were found positive with subtype H5 (accounting for 91.66\%). Similar to the situation in 2014, during the months of January, May, June, July, August, September, October, and November, no samples were available for testing. This result was similar to that of 2014 .

\subsection{Avian Influenza A Viruses Subtype H5N1 on Poultry Sold at Local Markets}

Cloacal and tracheal swab samples were collected of the chicken sold at periodic markets (there is a market day every five day): Gieng Vuong market (Lang Son city); Met market (Huu Lung district); Bac Son market (Bac Son district). In each periodic market, samples were collected from 60 chickens. The samples were then grouped into 12 composite swab samples. The results are presented in Table 6 .

Table 6 shows that: In Gieng Vuong market, 17 of the samples was found positive with avian influenza A viruses (accounting for 28.3\%); 7 samples were positive with 
subtype H5 (accounting for 11.66\%); 3 samples were positive with subtype N1 (accounting for 5\%). At Met market, 8 samples were positive with avian influenza A viruses (accounting for 13.33\%); positive with subtype H5 2 samples (accounting for $3.33 \%$ ); but none were found positive with subtype N1. At Bac Son market, 3 of the samples were found positive with avian influenza A viruses $(5 \%) ; 2$ were found positive with subtype $\mathrm{H} 5$ and 2 were found positive with subtype N1 (accounting for 3.33\%).

Table 6. Avian influenza A viruses, subtype H5N1 on chicken at the periodic markets.

\begin{tabular}{|c|c|c|c|c|c|c|c|c|}
\hline \multirow{3}{*}{ Periodic markets in } & \multirow{3}{*}{ No. of samples (Composite swabs) } & \multirow{3}{*}{ Negative } & \multicolumn{6}{|c|}{ Results } \\
\hline & & & \multicolumn{2}{|c|}{ Type A } & \multicolumn{2}{|c|}{ Subtype H5 } & \multicolumn{2}{|c|}{ Subtype N1 } \\
\hline & & & $(+)$ & $\%$ & $(+)$ & $\%$ & $(+)$ & $\%$ \\
\hline Met & 60 & 52 & 8 & 13.33 & 0 & 0 & 0 & 0 \\
\hline Bac Son & 60 & 57 & 3 & 5 & 2 & 3.33 & 2 & 3.33 \\
\hline Total & 180 & 152 & 28 & 15.55 & 9 & 5 & 5 & 2.77 \\
\hline
\end{tabular}

In 2014 and 2015, 28 out of 180 cloacal and tracheal swab samples of the chicken sold at the periodic markets in Lang Son province were found positive with avian influenza A viruses (accounting for 15.55\%); of which 9 were found positive with subtype H5 (accounting for 5\%); and 5 were found positive with subtype N1 (accounting for 2.77\%). This result was similar to that of [3], [9], [13].

\subsection{Avian Influenza Viruses Subtype H5N1 on the Ducks Sold at the Border Markets}

Cloacal and tracheal swab samples were collected of the ducks sold at 03 periodic markets in the border of Lang Son province (there is a market day every five day), including Dong Dang market (Cao Loc district), That Khe market (Trang Dinh district), and Loc Binh market (Loc Binh district). In each periodic market, samples were collected from 30 ducks. The samples were then grouped into 06 composite swab samples. The results are presented in table 7.

Table 7. Avian influenza A viruses, subtype H5N1 on the ducks sold at border markets.

\begin{tabular}{|c|c|c|c|c|c|c|c|c|}
\hline \multirow{3}{*}{ Border markets } & \multirow{3}{*}{ No. of samples (Composite swabs) } & \multirow{3}{*}{ Negative } & \multicolumn{6}{|c|}{ Results } \\
\hline & & & \multicolumn{2}{|c|}{ Type A } & \multicolumn{2}{|c|}{ Subtype H5 } & \multicolumn{2}{|c|}{ Subtype N1 } \\
\hline & & & $(+)$ & $\%$ & $(+)$ & $\%$ & $(+)$ & $\%$ \\
\hline Loc Binh & 42 & 39 & 3 & 7.14 & 0 & 0 & 0 & 0 \\
\hline Dong Dang & 42 & 32 & 10 & 23.8 & 5 & 11.9 & 4 & 9.52 \\
\hline That Khe & 42 & 38 & 4 & 9.52 & 1 & 2.38 & 1 & 2.38 \\
\hline Total & 126 & 109 & 17 & 13.49 & 6 & 4.76 & 5 & 3.96 \\
\hline
\end{tabular}

Table 7 shows that: At the periodic markets in Loc Binh, 3 composite swab samples were found positive with avian influenza A viruses (accounting for 7.14\%) but none were found positive with subtype $\mathrm{H} 5$ and N1. At the periodic markets in Dong Dang, 10 of the cloacal and tracheal swab samples from the ducks (composite samples) were found positive with avian influenza A viruses; of which 5 were found positive with subtype H5 (accounting for 11.9\%), 4 were found positive with subtype N1 (accounting for 3.96\%). At the periodic markets in That Khe, 4 were found positive with avian influenza A viruses (accounting for 9.52\%); of which 1 was found positive with subtype H5 and another sample was found positive with subtype N1 (accounting for $2.38 \%$ ).
In 2015, 17 out of 126 cloacal and tracheal swab samples of the ducks sold at 3 border markets in Lang Son province were found positive with avian influenza A viruses (accounting for 13.49\%); of which 6 were found positive with subtype H5 (accounting for 4.76\%); and 5 were found positive with subtype N1 (accounting for 3.96\%).

\subsection{Avian Influenza A Viruses Subtype H5N1 in the Environmental Matters at Poultry Selling Points}

Environmental swab samples (of waste and fecal matter) collected at poultry selling points were examined to detect the prevalence of avian influenza A viruses (H5N1). The results are presented in table 8 .

Table 8. Avian influenza A viruses, subtype H5N1 in environmental swab samples at poultry selling points.

\begin{tabular}{lllllll}
\hline \multirow{2}{*}{ Markets } & No. of samples & Results & & & \\
\cline { 3 - 6 } & & Type A & \% & H5 & \% & N1 \\
\hline Stage 1 & 32 & 2 & 6.25 & 2 & 0.25 & 0 \\
Na Duong & 32 & 1 & 3.12 & 0 & 0 & 0 \\
Gieng Vuong & 32 & 3 & 9.37 & 0 & 0 & 0 \\
Hoi Hoan & 32 & 2 & 6.25 & 0 & 0 & 0 \\
Dong Dang & & & & & 0 \\
Stage 2 & & & & \\
\hline
\end{tabular}




\begin{tabular}{llllllll}
\hline \multirow{2}{*}{ Markets } & \multirow{2}{*}{ No. of samples } & Results & & & & \\
\cline { 3 - 7 } & & Type A & $\mathbf{\%}$ & H5 & \% & N1 & \% \\
\hline Na Duong & 32 & 2 & 6.25 & 1 & 3.12 & 0 & 0 \\
Gieng Vuong & 32 & 3 & 9.37 & 1 & 3.12 & 0 & 0 \\
Hoi Hoan & 32 & 1 & 3.12 & 1 & 3.12 & 0 & 0 \\
Dong Dang & 32 & 2 & 6.25 & 0 & 0 & 0 & 0 \\
Stage 3 & & & & & & & 0 \\
Na Duong & 32 & 4 & 12.5 & 0 & 0 & 0 \\
Gieng Vuong & 32 & 2 & 6.25 & 1 & 3.12 & 0 \\
Hoi Hoan & 32 & 3 & 9.37 & 0 & 0 & 0 \\
Dong Dang & 32 & 2 & 6.25 & 0 & 0 & 0 & 0 \\
Total & 288 & 27 & 9.37 & 6 & 2.08 & 0 & 0 \\
\hline
\end{tabular}

Table 8 shows that: After conducting three independent stages of studying on poultry fecal matter and waste collected at the markets in Lang Son province, including $\mathrm{Na}$ Duong, Gieng Vuong, Hoi Hoan, and Dong Dang markets, 288 swab samples of waste matter and poultry fecal matter (chicken and ducks) were collected. Of which, 27 were found positive with avian influenza A viruses (accounting for 9.37\%); 6 were found positive with subtype $\mathrm{H} 5$ (accounting for $2.08 \%$ ); but none were found positive with subtype N1. This finding was similar to that of [9], [13]. The results also reflected the prevalence of avian influenza A viruses subtype $\mathrm{H} 5 \mathrm{~N} 1$ on the chicken and ducks illegally imported and sold at the local markets, border markets are presented in tables 5, 6, and 7 .

\subsection{Avian Influenza A Viruses Subtype H5N6 on Poultry at the Markets and Poultry Buying Points}

Besides the prevalence of avian influenza A viruses (H5N1) on the commercial poultry, the prevalence of avian influenza A viruses (H5N6) on chicken and ducks sold at border markets in 2014 and 2015 was also studied. The results are presented in Table 9.

Table 9. Avian influenza A viruses, subtype H5N6 on chicken and ducks at border markets.

\begin{tabular}{|c|c|c|c|c|c|c|c|c|c|}
\hline \multirow{3}{*}{ Markets } & \multirow{3}{*}{\multicolumn{2}{|c|}{ No. of samples (Composite swabs) }} & \multirow{3}{*}{ Negative } & \multicolumn{6}{|c|}{ Results } \\
\hline & & & & \multicolumn{2}{|c|}{ Type A } & \multicolumn{2}{|c|}{ Subtype H5 } & \multicolumn{2}{|c|}{ Subtype N6 } \\
\hline & & & & $\mathbf{A}$ & $\%$ & H5 & $\%$ & N6 & $\%$ \\
\hline \multicolumn{10}{|l|}{ In 2014} \\
\hline Na Duong & \multicolumn{2}{|l|}{24} & 20 & 4 & 16.6 & 2 & 8.3 & 2 & 8.3 \\
\hline $\mathrm{Na}$ Sam & \multicolumn{2}{|l|}{24} & 23 & 1 & 4.2 & 1 & 4.2 & 1 & 42 \\
\hline $\begin{array}{l}\text { Total } \\
\text { In } 2015\end{array}$ & \multicolumn{2}{|l|}{48} & 43 & 5 & 10.4 & 3 & 6.25 & 3 & 6.25 \\
\hline \multirow{2}{*}{ Markets } & \multicolumn{5}{|c|}{ No. of samples (Composite swabs) } & \multicolumn{4}{|c|}{ Results } \\
\hline & $\mathrm{HH}$ & $\mathrm{NU}$ & NT & $\mathrm{CT}$ & $\mathrm{P}$ & $(-)$ & A & H5 & N6 \\
\hline Hoi Hoan & 36 & 3 & 6 & 6 & 3 & 37 & 18 & 1 & 1 \\
\hline Dong Dang & 36 & 3 & 6 & 6 & 3 & 24 & 13 & 2 & 1 \\
\hline Gieng Vuong & 36 & 3 & 6 & 6 & 3 & 36 & 30 & 5 & 2 \\
\hline Na Duong & 36 & 3 & 6 & 6 & 3 & 41 & 17 & 1 & 1 \\
\hline Total & 144 & 12 & 24 & 24 & 12 & 138 & 78 & 9 & 5 \\
\hline
\end{tabular}

Notes: H: Cloacal and tracheal swab samples; NU: Samples of poultry drinking water collected from water containers; NT: Samples of water collected from the cage floors; P: Samples of fecal matter from chicken; CT: Samples of waste matter.

Table 9 shows that: In 2014, 48 composite cloacal and tracheal swab samples of chicken and ducks at two district border markets of $\mathrm{Na}$ Duong and $\mathrm{Na}$ Sam, and 10.4\% were found positive with avian influenza A viruses; of which, $6.25 \%$ belong to subtype $\mathrm{H} 5$; and $6.25 \%$ belong to subtype N6. In 2015, 216 samples were collected from the markets of Hoi Hoan, Dong Dang, Gieng Vuong and Na Duong, of which, there were 144 cloacal and tracheal swab samples, 12 samples of poultry drinking water collected from poultry drinking water containers, 24 samples of waste water; 24 samples of waste matter; and 12 samples of fecal matter from chicken and ducks. The results show that 78 samples were found positive with avian influenza A viruses (accounting for $36.1 \%$ ); 9 were found positive with subtype H5 (accounting for $4.1 \%$ ); and 5 were found positive with subtype N6 (accounting for $2.3 \%$ ).
The results show the prevalence of avian influenza viruses poultry on commercial chicken and ducks sold at border markets in Lang Son. Besides type A, subtype H5N1, there were also avian influenza A viruses, subtype H5N6. The results were similar to the contents of the report on avian influenza virus situation in Vietnam in general and in Northern mountainous provinces of Vietnam in particular. This finding was similar to that of [1].

\subsection{Avian Influenza A Viruses, H7N9 on Poultry at the Markets and Collection Points}

The prevalence of avian influenza A viruses, subtype H7N9 on poultry (chicken and ducks) sold at the markets (local and border markets) and poultry buying points in Lang Son province (in 2014 and 2015) was examined. The results are presented in tables 10,11 and 12 . 
Table 10. Avian influenza A viruses, subtype H7N9 in environmental samples at poultry buying points and markets (Sample collecting frequency: every 03 days, in 10 stages).

\begin{tabular}{|c|c|c|c|c|c|c|c|c|c|}
\hline \multirow{2}{*}{ Areas (Poultry buying points or markets) } & \multicolumn{5}{|c|}{ No. of samples (Composite swabs) } & \multicolumn{4}{|c|}{ Results } \\
\hline & HH & NU & NT & CT & $\mathbf{P}$ & $(-)$ & $\mathbf{A}$ & H7 & N9 \\
\hline Dong Mo & 70 & 8 & 4 & 3 & 55 & 129 & 11 & - & - \\
\hline Ky Lua & 70 & 19 & 21 & 3 & 27 & 110 & 30 & - & - \\
\hline Dong Kinh & 70 & 20 & 20 & 20 & 10 & 108 & 32 & - & - \\
\hline Chi Lang & 70 & 20 & 20 & 20 & 10 & 134 & 6 & - & - \\
\hline Hoang Van Thu & 70 & 20 & 20 & 20 & 10 & 115 & 25 & - & - \\
\hline Tran Khanh Du & 70 & 20 & 20 & 20 & 10 & 120 & 20 & - & - \\
\hline Loc Binh & 70 & - & - & 20 & 50 & 115 & 25 & - & - \\
\hline Cao Loc & 70 & 20 & 19 & 18 & 13 & 87 & 53 & - & - \\
\hline Chi Lang & 70 & 20 & 20 & 19 & 11 & 67 & 73 & - & - \\
\hline Ba Trieu & 70 & 20 & 20 & 20 & 10 & 86 & 54 & - & - \\
\hline Nhan Ly & 70 & 19 & 19 & 20 & 12 & 136 & 4 & - & - \\
\hline Hop Thanh & 70 & 20 & 20 & 20 & 10 & 70 & 70 & - & - \\
\hline Tam Thanh & 70 & 20 & 18 & 19 & 13 & 91 & 49 & - & - \\
\hline Dong Dang & 70 & 20 & 20 & 20 & 10 & 129 & 11 & - & - \\
\hline Vuon Sai & 70 & 20 & 20 & 20 & 10 & 126 & 14 & - & - \\
\hline Total & 1,050 & 266 & 261 & 262 & 261 & 1,623 & 477 & - & - \\
\hline
\end{tabular}

Notes: HH: Cloacal and tracheal swab samples; NU: Samples of poultry drinking water collected from water containers; NT: Samples of water collected from the cage floors; P: Samples of fecal matter from chicken; CT: Samples of waste matter.

Table 10 shows that 3,723 samples were examined, including 1,050 cloacal and tracheal swab samples, 266 samples of poultry drinking water collected from water containers, 261 samples of waste water, 262 samples of waste matter, and 261 samples of fecal matter from chicken and ducks sold at the markets and poultry buying points in Lang
Son at the sample collection frequency of once every three days in ten stages. The results showed that 477 were found positive with avian influenza A viruses. However, none were found positive with subtype H7 and subtype N9. This result was similar to that of [9], [14], [15].

Table 11. Avian influenza A viruses, H7N9 on chicken at local markets and poultry buying points (Sample collecting frequency: once every ten days).

\begin{tabular}{|c|c|c|c|c|c|c|c|c|c|}
\hline \multirow{2}{*}{ Areas (Poultry buying points or markets) } & \multicolumn{5}{|c|}{ Types of samples } & \multicolumn{4}{|c|}{ Results } \\
\hline & HH & NU & NT & CT & $\mathbf{P}$ & $(-)$ & $\mathbf{A}$ & H7 & N9 \\
\hline Hoang Van Thu & 42 & 12 & 12 & 12 & 6 & 66 & 18 & - & - \\
\hline Chi Lang & 42 & 12 & 12 & 12 & 6 & 73 & 11 & - & - \\
\hline Hop Thanh & 42 & 12 & 12 & 12 & 6 & 55 & 29 & - & - \\
\hline Ky Lua & 42 & 12 & 12 & 12 & 6 & 61 & 23 & - & - \\
\hline Dong Kinh & 42 & 12 & 12 & 12 & 6 & 72 & 12 & - & - \\
\hline Chi Lang & 42 & 12 & 12 & 12 & 6 & 65 & 19 & - & - \\
\hline Dong Dang & 42 & 0 & 14 & 10 & 18 & 63 & 21 & - & - \\
\hline Vuon Sai & 42 & 0 & 13 & 12 & 17 & 68 & 16 & - & - \\
\hline Dong Mo & 42 & 12 & 12 & 12 & 6 & 65 & 19 & - & - \\
\hline Lang Son city 1 & 42 & 12 & 12 & 12 & 6 & 67 & 17 & - & - \\
\hline Ba Trieu & 42 & 12 & 12 & 12 & 6 & 58 & 26 & - & - \\
\hline Cao Loc & 42 & 12 & 12 & 12 & 6 & 65 & 19 & - & - \\
\hline Lang Son city 2 & 42 & 12 & 12 & 12 & 6 & 82 & 2 & - & - \\
\hline Total & 630 & 155 & 183 & 178 & 114 & 998 & 262 & - & - \\
\hline
\end{tabular}

Table 11 presents the results of the examination of the prevalence of Avian influenza A viruses, H7N9 on chicken sold at local markets and poultry buying points with the frequency of sample collection of once every ten days. The results show that: In total, there were 1,260 samples examined including 630 cloacal and tracheal swab samples, 155 samples of poultry drinking water collected from water containers, 183 samples of waste water, 178 samples of waste matter and 114 samples of poultry fecal matter. The results show that 262 of the samples were found positive with avian influenza A viruses (accounting for 20.7\%), and the results are similar to those presented in Table 7, but none were found positive with subtypes H7 or N9. This result was similar to that of [14], [15]. 
Table 12. Avian influenza A viruses, H7N9 on poultry (chicken and ducks) at border markets (Sample collecting frequency: once every 15 days).

\begin{tabular}{|c|c|c|c|c|c|c|c|c|c|}
\hline \multirow{2}{*}{ Areas (Markets) } & \multicolumn{5}{|c|}{ No. of samples (Composite swabs) } & \multicolumn{4}{|c|}{ Results } \\
\hline & HH & NU & NT & CT & $\mathbf{P}$ & $(-)$ & $\mathbf{A}$ & H7 & N9 \\
\hline Na Duong & 36 & 6 & 6 & 3 & 3 & 37 & 17 & - & - \\
\hline Gieng Vuong & 36 & 6 & 6 & 3 & 3 & 24 & 30 & - & - \\
\hline Hoi Hoan & 36 & 6 & 6 & 3 & 3 & 36 & 18 & - & - \\
\hline Dong Dang & 36 & 6 & 6 & 3 & 3 & 41 & 15 & - & - \\
\hline Total & 144 & 24 & 24 & 12 & 12 & 138 & 78 & - & - \\
\hline
\end{tabular}

Table 12 shows that: 216 samples were collected and examined, including 144 cloacal and tracheal swab samples, 24 samples of poultry drinking water collected from water containers, 24 waste water samples, 12 samples of waste matter fecal matter. Of which, 262 were found positive with avian influenza $\mathrm{A}$ viruses (accounting for $36.1 \%$ ), which was similar to the results shown in table 10 and table 11 , but none were found positive with subtype H7 or subtype N9.

The results of this study are similar to the report of the Department of Animal Health. In 2015 China recorded 225 cases of A/H7N9 viruses infection on human, with 94 dead. Thus, since March 2013 H7N9 viruses infection on human continuously occurred in China. This finding was similar to that of [3], [5], [14], [15]. The results show that there was no prevalence of avian influenza viruses subtype H7N9 on commercial poultry sold at border and local markets in Lang Son.

\subsection{Virulence Assessment on Laboratory Chicken (IVPI)}

An experiment was carried out to determine the virulence level (Intravenous Pathogenicity Index) on six-week chicken which had been identified as having no antibody against avian influenza A viruses subtype H5N1. The results are presented in Table 13.

Table 13. Virulence Assessment on laboratory chicken (IVPI).

\begin{tabular}{|c|c|c|c|c|c|c|c|c|c|c|c|c|}
\hline \multirow{2}{*}{ Clinical symptoms } & \multicolumn{10}{|c|}{ Monitoring days } & \multirow{2}{*}{ Total } & \multirow{2}{*}{ Point } \\
\hline & N1 & N2 & N3 & N4 & N5 & N6 & N7 & N8 & N9 & N10 & & \\
\hline \multicolumn{13}{|l|}{ Virus group 1} \\
\hline Normal & 6 & 6 & 6 & 6 & 6 & 6 & 6 & 6 & 6 & 6 & $60 \times 0$ & 0 \\
\hline Sick & 1 & 0 & 0 & 0 & 0 & 0 & 0 & 0 & 0 & 0 & $1 \times 1$ & 1 \\
\hline Very sick & 1 & 0 & 0 & 0 & 0 & 0 & 0 & 0 & 0 & 0 & $1 \times 2$ & 2 \\
\hline Dead & 2 & 4 & 4 & 4 & 4 & 4 & 4 & 4 & 4 & 4 & $38 \times 3$ & IVPI $=2.92$ \\
\hline \multicolumn{13}{|l|}{ Virus group 2} \\
\hline Normal & 7 & 7 & 7 & 7 & 7 & 7 & 7 & 7 & 7 & 7 & $70 \times 0$ & 0 \\
\hline Sick & 1 & 0 & 0 & 0 & 0 & 0 & 0 & 0 & 0 & 0 & $1 \times 1$ & 1 \\
\hline Very sick & 1 & 0 & 0 & 0 & 0 & 0 & 0 & 0 & 0 & 0 & $1 \times 2$ & 2 \\
\hline Dead & 1 & 3 & 3 & 3 & 3 & 3 & 3 & 3 & 3 & 3 & $28 \times 3$ & IVPI=2.9 \\
\hline \multicolumn{13}{|l|}{ Virus group 3} \\
\hline Normal & 6 & 6 & 6 & 6 & 6 & 6 & 6 & 6 & 6 & 6 & $60 \times 0$ & 0 \\
\hline Sick & 1 & 0 & 0 & 0 & 0 & 0 & 0 & 0 & 0 & 0 & $1 \times 1$ & 1 \\
\hline Very sick & 1 & 0 & 0 & 0 & 0 & 0 & 0 & 0 & 0 & 0 & $1 \times 2$ & 2 \\
\hline Dead & 2 & 4 & 4 & 4 & 4 & 4 & 4 & 4 & 4 & 4 & $38 \times 3$ & IVPI $=2.92$ \\
\hline \multicolumn{13}{|l|}{ Virus group 4} \\
\hline Normal & 6 & 6 & 6 & 6 & 6 & 6 & 6 & 6 & 6 & 6 & $60 \times 0$ & 0 \\
\hline Sick & 1 & 0 & 0 & 0 & 0 & 0 & 0 & 0 & 0 & 0 & $1 \times 1$ & 1 \\
\hline Very sick & 1 & 0 & 0 & 0 & 0 & 0 & 0 & 0 & 0 & 0 & $1 \times 2$ & 2 \\
\hline Died & 2 & 4 & 4 & 4 & 4 & 4 & 4 & 4 & 4 & 4 & $38 \times 3$ & $\mathrm{IVPI}=2.92$ \\
\hline \multicolumn{13}{|l|}{ Controlled group } \\
\hline Normal & 10 & 10 & 10 & 10 & 10 & 10 & 10 & 10 & 10 & 10 & $100 \mathrm{x} 0$ & 0 \\
\hline Sick & 0 & 0 & 0 & 0 & 0 & 0 & 0 & 0 & 0 & 0 & $0 \times 1$ & 0 \\
\hline Very sick & 0 & 0 & 0 & 0 & 0 & 0 & 0 & 0 & 0 & 0 & $0 \times 2$ & 0 \\
\hline Dead & 0 & 0 & 0 & 0 & 0 & 0 & 0 & 0 & 0 & 0 & $0 \times 3$ & 0 \\
\hline
\end{tabular}

Notes:

IVPI: intravenous pathogenicity index

Viruses group 1: H5N1 viruses strain prevalent on illegally imported chicken from China to Vietnam

Viruses group 2: H5N1 viruses strain prevalent on chicken sold at local periodic markets in Lang Son province

Viruses group 3: H5N1 viruses strain prevalent on the ducks at periodic markets in the border areas between Vietnam and China

Viruses group 4: H5N6 viruses strain prevalent on the ducks at periodic markets in the border areas between Vietnam and China

Controlled group: The injecting fluid did not contain viruses

N: Day 
Table 13 shows that in compared with the controlled group, all of the laboratory chickenshowed symptoms of being sick within 1 or 2 days after being infected with the strains of avian influenza A viruses/H5N1 and type A/H5N6 isolated. The intravenous pathogenicity index (IVPI) of the virus strains was measured at the levels ranging from 2.9 (the viruses strain found on the chicken sold at local periodic markets) to 2.92 (the viruses strain $\mathrm{H} 5 \mathrm{~N} 1$ and $\mathrm{H} 5 \mathrm{~N} 6$ isolated on illegally imported chicken from China and ducks sold at border markets in Lang Son). The results were similar to the analysis of [3], [7], [8], [9], [12], and [15] in their studies on sickness causing characteristics of avian influenza viruses on poultry.

\section{Conclusion}

- The chicken illegally imported from China to Lang Son, Viet Nam were found with avian influenza A viruses, subtype H5N1, with the ratios ranging from $3.93 \%$ to 6 . $43 \%$ with type A, from $3.18 \%$ to $3.78 \%$ with subtype $\mathrm{H} 5$, and from $0.37 \%$ to $3.40 \%$ with subtype N1.

- The chicken sold at local periodic markets in Lang Son province were found with avian influenza A viruses, subtype $\mathrm{H} 5 \mathrm{~N} 1$, including $15.55 \%$ with type A, $5 \%$ with subtype $\mathrm{H} 5$, and $2.77 \%$ with subtype $\mathrm{N} 1$.

- The ducks sold at border markets between Lang Son and China which were found with avian influenza A viruses, subtype $\mathrm{H} 5 \mathrm{~N} 1$, including $13.49 \%$ with type A, $4.76 \%$ with subtype $\mathrm{H} 5$, and $3.96 \%$ with subtype $\mathrm{N} 1$.

- The commercial chicken and ducks sold at border markets Lang Son were found with avian influenza A viruses, subtype H5N6. In 2014, 10.4\% were found positive with avian influenza $\mathrm{A}$ viruses, with $6.25 \%$ belong to subtype $\mathrm{H} 5$ and $6.25 \%$ belong to subtype N6; In $2015,36.1 \%$ were found with avian influenza A viruses, $4.1 \%$ were found with subtype $\mathrm{H} 5$, and $2.3 \%$ were found with subtype N6.

- The study in 2014 and 2015 shows that in Lang Son there was no prevalence of subtype H7N9 on commercial poultry (chicken and ducks) sold at the border and local markets.

- The isolated amniotic fluid samples of the embryos cultured with avian influenza A viruses, subtype H5N1 and type A, subtype H5N6 in Lang Son were found with virulence (causing sicknesses) which caused the deaths of the laboratory chicken within one or two days after being infected with the viruses, with the Intravenous Pathogenicity Index (IVPI) ranging from 2.9 to 2.92 .

\section{Acknowledgments}

In making this project possible, we would like to express our special gratitude to Lang Son Sub Department of Animal Health, National Centre for Veterinary Diagnosis, and Regional Animal Health Office No. II for their technical support and Centers for Disease Control and Prevention (CDC, USA) for their technical, facility, and financial support through Food and Agriculture Organization (FAO) in implementing "Vietnam Avian and Human Influenza Control and Preparedness Project in Lang Son province" (VAHIP).

\section{References}

[1] Beuy Joob, Wiwanitkit Viroj (2016). H5N6 influenza viruses infection, the newest influenza. Asian Pacific Journal of Tropical Biomedicine. Volume 5, Issue 6, June 2015, Pages 434-437.

[2] Bui Q. A, Van D. K (2004). Avian influenza viruses diseases: Prevalence, Diagnosis, and Control. Journal of Animal Health Science and Technology, (3), pages 69-75.

[3] Cowling BJ, Jin L, Lau EH, Liao Q, Wu P, Jiang $\mathrm{H}$, Comparative epidemiology of human infections with avian influenza A H7N9 and $\mathrm{H} 5 \mathrm{~N} 1$ viruses in China: a populationbased study of laboratory-confirmed cases. Lancet. 2013; 382: 129-37.

[4] Dung N. T (2008), Some characteristics of avian influenza viruses H5N1, Journal of Animal Health Science and Technology, (4), pages 80-86.

[5] Han J, Liu J, Wang L, Zhang P, Liu G, Lan K (2014). Persistent detection of avian influenza A/H7N9 virus among poultry in Huzhou City, China, in the summer of 2013. Int J Infect Dis. 2014; 26: 72-5.

[6] Leung YH, Lau EH, Zhang LJ, Guan Y, Cowling BJ, Peiris JS (2012). Avian influenza and ban on overnight poultry storage in live poultry markets, Hong Kong. Emerg Infect Dis. 2012; 18: 1339-41.

[7] Mary J. Pantin-Jackwood, Jenny Pfeiffer, To L. T, Tung N and David Suarez (2008). Virulence level of the highly virulent avian influenza viruses H5N1 chicken and ducks in Vietnam, International Conference on Studying for Policy Development for avian influenza viruses prevention and control, Department of Animal Health, 2008.

[8] Monne I, Fusaro A, Nelson MI, Bonfanti L, Mulatti P, Hughes $\mathrm{J}$ (2014). Emergence of highly pathogenic avian influenza viruses from a low-pathogenic progenitor. J Virol. 2014; 88: 4375-88.

[9] Kosuke Okuya, Toshiko Kawabata, Kiori Nagano, Kyoko Tsukiyama-Kohara, Isamu Kusumoto, Kozo Takase, Makoto Ozawa (2015). Isolation and characterization of influenza $A$ viruses from environmental water at an overwintering site of migratory birds in Japan. Archives of Virology. Volume 160, Issue 12, pp 3037-3052.

[10] Office International des Epizootics (OIE), 2014.

[11] Pantin-Jackwood MJ, Miller PJ, Spackman E, Swayne DE, Susta L, Costa-Hurtado M (2014). Role of poultry in the spread of novel H7N9 influenza virus in China. J Virol. 2014; 88: 5381-90.

[12] Shankar B. P, R. N. S. Gowda, B. H. Manjunath Prabhu, B. Pattnaik, S. Nagarajan, S. S. Patil and H. K. Pradhan (2009). Assessment of Pathogenic Potential of Two Indian H5N1 Highly Pathogenic Avian influenza Viruses Isolates by Intravenous Pathogenicity Index Test. International Journal of Poultry Science 8 (3): 283-290. 
[13] Vries, E., H. Guo, M. Dai, P. J. Rottier, F. J. van Kuppeveld and C. A. de Haan (2015). Rapid Emergence of Highly Pathogenic Avian influenza Subtypes from a Subtype H5N1 Hemagglutinin Variant. Emerg Infect Dis 21 (5): 842-846.

[14] Jun Yuan, Eric H. Y. Lau, Kuibiao Li, Y. H. Connie Leung, Zhicong Yang, Caojun Xie, Yufei Liu, Yanhui Liu, Xiaowei Ma, Jianping Liu, Xiaoquan Li, Kuncai Chen, Lei Luo, Biao Di, Benjamin J. Cowling, Xiaoping Tang, Gabriel M. Leung, Ming Wang, and Myoung-don Oh (2014). Effect of Live Poultry Market Closure on Avian influenza A (H7N9) Viruses
Activity in Guangzhou, China, 2014. Volume 21, Number 10. October 2015.

[15] Wang X, Fang S, Lu X, Xu C, Cowling BJ, Tang X (2014). Seroprevalence to avian influenza A (H7N9) virus among poultry workers and the general population in southern China: a longitudinal study. Clin Infect Dis. 2014; 59: e76-83.

[16] WHO (2015). Risk assessment of Human infections with avian influenza $A$ (H7N9) viruses. Summary of surveillance and investigation findings. 23 February 2015. 